\title{
GLI ITALIANISMI ENOGASTRONOMICI RECENTI NELLA LINGUA INGLESE: UN'ANALISI SUI CORPORA
}

\author{
Daniele Della Pietra ${ }^{1}$
}

La lingua italiana ha da sempre ricoperto un ruolo determinante nel panorama linguistico europeo e internazionale, esercitando un notevole influsso sia sulle lingue romanze sia su alcune lingue germaniche come il tedesco o l'inglese. Basti pensare agli innumerevoli italianismi presenti nelle diverse lingue, favoriti soprattutto dal fenomeno della globalizzazione.

L'articolo muove dall'intento di identificare e analizzare i lessemi di origine italiana presenti nella lingua inglese in ambito enogastronomico, entrati a far parte negli ultimi anni della lingua veicolare per eccellenza, sfruttando, come punto di partenza, l'Oxford English Dictionary (OED) e come termine di paragone il Dižionario di italianismi in francese, inglese, tedesco (DIFIT), una notevole opera lessicografica che prende in esame più di 5000 italianismi.

La ricerca è stata resa possibile grazie all'utilizzo di tre corpora ovvero enTenTen $15^{2}$, presente all'interno della piattaforma Sketch Engine, e due corpora ricavati dal New York Times Cooking e da BBC Good Food, le sezioni di cucina rispettivamente del quotidiano statunitense New York Times e dell'ente di informazione britannico BBC, i quali permettono di valutare la presenza degli italianismi nell'inglese americano e nell'inglese britannico.

\section{GLI ITALIANISMI}

Gli italianismi costituiscono motivo di interesse per almeno due ragioni: la prima è evidentemente di tipo linguistico: il passaggio di una parola da una lingua ad un'altra non solo dall'italiano, quindi - provoca fenomeni di adattamento, di mutamento, di reazione. Sul piano fonetico, ad esempio, la necessità di accogliere in un sistema assai difforme dall'italiano fonemi italiani può portare a vistosi adattamenti: in giapponese allegro e salame divengono areguro e sarami, nell'arabo parlato in Egitto partitura e pasta, bartituura e basta, in finlandese fuga e medaglia, funga e mitali. L'altra ragione di interesse è di tipo culturale nel senso più ampio del termine: gli italianismi raccontano ciò che dell'Italia e degli italiani è parso all'estero notevole o tipico a qualche titolo, o riflettono aspetti della cultura, invenzioni o prodotti, che hanno avuto la loro origine o la loro fortuna in Italia, tanto da diffondersi insieme alla parola italiana che li designava (Rossi, 2009).

L'italianismo è una voce lessicale, locuzione o costruzione sintattica propria della lingua italiana introdotta in altra lingua, o in un dialetto (Stammerjohann, 2010). Gli italianismi

\footnotetext{
${ }^{1}$ Università degli Studi Internazionali di Roma - UNINT.

${ }^{2} \mathrm{Il}$ corpus enTenTen15 è attualmente uno dei più estesi corpora linguistici presenti su Sketch Engine e appartiene alla famiglia di corpora TenTen, la quale comprende 38 lingue fra cui anche l'italiano. In esso sono presenti 13 miliardi di occorrenze ricavate da testi presi dal web durante l'anno 2015.

La piattaforma Sketch Engine è consultabile online al link: https://www.sketchengine.eu/.
} 
sono principalmente sostantivi e, in piccola quantità, aggettivi (Stammerjohann, 2013). Derivano, spesso, dal prestigio linguistico dell'italiano che per influsso ha arricchito lessicalmente le lingue romanze (questo vale per esempio per lo spagnolo, il portoghese, il rumeno) $)^{3}$.

Anche alcune lingue di derivazione germanica, slava oppure uralica presentano numerosi italianismi, a dimostrazione della grande capacità di permeazione linguistica dell'italiano: per esempio nel finlandese o nello sloveno ${ }^{4}$.

Innumerevoli, infine, gli studi sulla presenza degli italianismi in lingua francese, inglese e tedesca catalogati e raccolti in maniera puntuale e precisa nel DIFIT, banca dati di notevole valore che intercetta e cataloga gli italianismi presenti nelle lingue sopracitate ed appartenenti ai più disparati ambiti ${ }^{5}$.

\subsection{Cenni storici sugli italianismi}

Gli italianismi riflettono i contatti, immediati o mediati, con la civiltà italiana e variano a seconda delle circostanze. I primi italianismi attestati sono del XII secolo e sono di origine dialettale, o sono a loro volta arabismi, passati in italiano sia direttamente dall'arabo, in quanto l'Italia aveva una funzione mediatrice tra l'Europa e il Levante, sia indirettamente attraverso la penisola iberica, per es. zucchero, marzapane, sorbetto, passati in molte lingue. I primi italianismi di origine indigena riflettono il dominio dell'Italia nei campi della marineria mediterranea, del commercio e dell'arte militare, prima che in quello della cultura letteraria e artistica (Stammerjohann, 2010).

Un esempio pratico dell'influsso legato al lessico marinaresco italiano, iniziato a diffondersi nelle lingue europee a partire dal XII secolo con le Repubbliche marinare, è dato da due lessemi: gondola e fregata. Per il primo termine, avremo in francese gondole, in inglese gondola, in spagnolo góndola, in tedesco gondel, in portoghese gôndola (Tomasin, 2010, 2011). Uno scenario simile si ripropone per la tipologia di nave militare denominata fregata: frégate in francese, frigate in inglese, fragata in spagnolo e in portoghese e infine in tedesco fregatte. Ugualmente importanti in questo periodo storico sono i termini militari, per es. soldato in albanese sol'dát, danese soldat, francese soldat, greco moderno soldádos, olandese soldaat, romeno soldat, spagnolo e portoghese soldado, tedesco Soldat ecc.

Tra il XIV e XV secolo, con la nascita delle prime banche e della finanza, iniziano a diffondersi in Europa termini legati all'ambito finanziario come è il caso riscbio e valuta, rintracciabili nelle lingue sopracitate; basti pensare all'inglese risk e value.

Il Rinascimento vede il primato italiano anche nel campo delle arti e dell'architettura, e di conseguenza una molteplicità di termini di tali ambiti penetrano nelle lingue d'Europa. Ł̀ il caso di lessemi come artista, chiaroscuro, fresco, schizzo, tempera, maniera, sveltezza; architetto, ordine (architettonico), bassorilievo, cannellatura, cavetto, cornicione, stucco (Motolese, 2012).

Insieme alle arti figurative e all'architettura fiorisce anche la musica lirica italiana, e di conseguenza anche il lessico annesso. Il primato italiano in campo musicale comporta che lessemi italiani o modellati sull'italiano entrino tra il Cinque e Settecento nelle grandi lingue di cultura, che musicisti stranieri (Haydn, Mozart, Gluck) adottino l'italiano per le loro composizioni e che il melodramma (opere di Metastasio e di altri) trionfi nelle corti e nei teatri d'Europa, facendo dell'italiano una vera e propria "lingua per la musica", sia nella Penisola che nei grandi stati europei. Con l'inizio dell'Ottocento l'opera lirica italiana

\footnotetext{
${ }^{3}$ Cfr. Ayala Simón (2002), Endruschat (2010), Dimitrescu (2003).

${ }^{4}$ Cfr. Pinomaa (2018), Ožbot (2008).

${ }^{5}$ Cfr. Cap 2.2.
} 
subisce la concorrenza delle rivali francese e tedesca e si attenua (ma non svanisce) il suo predominio, a causa della crescente abitudine di servirsi di libretti scritti nella lingua del paese ospitante.

È rivelatrice della perdurante fortuna della nostra lingua, ancora ai nostri giorni, la padronanza dell'italiano che dimostrano cantanti e musicisti stranieri, non solo nella professione ma anche nella vita quotidiana. In moltissimi casi hanno diffusione internazionale italianismi non adattati. Si pensi alla terminologia inglese (e internazionale) di movimenti musicali (adagio, andante, allegro, accelerando, crescendo, forte, fortissimo, lento, maestoso); o di composizioni (ballabile, concertino, sinfonietta); o di protagonisti dell'opera (baritono, basso, contralto, mezzosoprano, tenorino); o di strumenti (pianoforte, pianola, tuba, viola, violoncello) (Coluccia, 2017).

A partire dalla seconda metà dell'Ottocento, un numero considerevole di italiani emigrano verso il continente americano in cerca di un futuro migliore, in particolar modo negli Stati Uniti. Molti di loro lavorano e contribuiscono alla crescita economica statunitense in maniera onesta, tuttavia vi è una minor parte che dà il via a una serie di comportamenti criminali che porteranno alla nascita della cosiddetta cosa nostra americana. Arrivano di conseguenza negli Stati Uniti, per poi diffondersi in tutto il mondo termini quali mafia e omertà, riscontrabili sia nelle lingue romanze che in quelle germaniche come prestiti non adattati, benché di ampia circolazione.

Dopo quello dell'arte, della pittura, della scultura e della musica, il campo semantico del cibo è quello che ha maggiormente rifornito la lingua inglese di nuovi termini di origine italiana, almeno secondo le cifre contenute nella seconda edizione dell'Oxford English Dictionary (Pinnavaia, 2006: 9).

La diffusione estera di alcuni lemmi è assai precoce (lasagne a partire dal XVI secolo; successivamente aleatico, panettone, pappardelle); ma la grande maggioranza è di epoca recente e recentissima, in collegamento con la crescente globalizzazione dei consumi: pizza (che pare sia la parola italiana più diffusa al mondo), pasta, spaghetti, cannelloni, cappuccino, espresso, mortadella, salame, tiramisù, e tanti altri si espandono in moltissime lingue dei cinque continenti, con vari adattamenti che talora modificano grafia, fonetica, morfologia e perfino semantica delle forme originarie (Coluccia, 2017: 482).

\subsection{Gastronimi nell'inglese americano}

L'ambito dell'enogastronomia è tra quelli che generano più italianismi nelle lingue straniere e in particolar modo in quella inglese. Come illustrato da Laura Pinnavaia (2006: 9) nella tabella 1 riportata di seguito, i lessemi di origine italiana legati all'enologia e alla gastronomia circolano e aumentano di numero dal Quattrocento fino a oggi, a testimonianza del notevole influsso culturale di matrice italiana dapprima sull'inglese d'Inghilterra e in seguito su quello americano.

Tabella 1. Lessemi di origine italiana legati all'enologia e alla gastronomia dal Quattrocento fino a oggi

\begin{tabular}{|c|c|c|c|c|}
\hline Periodo & Alimenti & Bevande & Arte Culinaria & Totale \\
\hline $1400-1450$ & 2 & & & 2 \\
\hline $1450-1500$ & & & & \\
\hline $1500-1550$ & 3 & 1 & & 4 \\
\hline $1550-1600$ & 2 & 1 & & 3 \\
\hline
\end{tabular}


(C) Italiano LinguaDue 2. 2021. D. Della Pietra, Gli italianismi enogastronomici recenti nella lingua inglese: un'analisi sui corpora

\begin{tabular}{|l|c|c|c|c|}
\hline \multicolumn{1}{|c|}{ Periodo } & Alimenti & Bevande & Arte Culinaria & Totale \\
\hline $1600-1650$ & 8 & 4 & 1 & 13 \\
\hline $1650-1700$ & 3 & 4 & 1 & 8 \\
\hline $1700-1750$ & 1 & 1 & & 2 \\
\hline $1750-1800$ & 6 & 2 & & 8 \\
\hline $1800-1850$ & 16 & 6 & 1 & 23 \\
\hline $1850-1900$ & 24 & 7 & & 31 \\
\hline $1900-1950$ & 47 & 9 & 2 & 58 \\
\hline $1950-2000$ & 41 & 9 & & 50 \\
\hline Totale & 153 & 44 & 5 & 202 \\
\hline
\end{tabular}

Durante la prima metà del XV secolo i primi gastronimi italiani, ovvero radish e rafiol, circolano in inglese $e^{6}$, e con il tempo si sono adattati oppure hanno generato altri termini. Il primo termine, derivante dall'italiano radice, presenta un notevole adattamento nella lingua inglese. Rafiol deriverebbe dall'omonima variante dell'italiano settentrionale dell'epoca per fare riferimento ai ravioli. Tuttavia, il lessema nel corso del tempo ha subito modificazioni e ha seguito il corso della medesima parola presente anche in italiano standard, di conseguenza andando a divenire intorno all'Ottocento, ravioli.

Se nel Quattrocento il fenomeno degli italianismi può sembrare governato dall'occasionalità, nei due secoli successivi gli italianismi enogastronomici diventano un fenomeno consolidato dovuto da una parte al Rinascimento, epoca fiorente per la cultura italiana, e dall'altra all'avvento del Grand Tour, un lungo viaggio formativo, con meta principale l'Italia, intrapreso dai giovani aristocratici europei, i quali avevano l'opportunità di venire a contatto con la lingua italiana e le sue varianti dell'epoca e di riportare nella propria lingua di origine numerosi lessemi appresi durante il soggiorno nella penisola. Di conseguenza nel XVI e XVII secolo entrano a far parte dell'inglese d'Inghilterra 28 ulteriori lessemi italiani legati all'ambito enogastronomico.

Nel Settecento il flusso di italianismi enogastronomici in inglese subisce un forte rallentamento dovuto all'ascesa economica e culturale dell'Inghilterra che, per tutta la durata del secolo, ricoprirà quel ruolo che era stato della penisola italica in epoca rinascimentale, andando a influenzare le altre culture europee anziché subirne il fascino. Nonostante tale situazione, l'inglese accoglie dieci nuovi termini, fra i quali lasagna e finocchio, ancora oggi in circolazione.

L'incremento degli italianismi enogastronomici in inglese si concretizza nell'Ottocento con la grande migrazione negli Stati Uniti, vi è quindi una crescita esponenziale di italianismi nell'inglese statunitense da un lato legato alla varietà linguistica utilizzata nel Regno Unito, dall'altro all'influsso degli emigrati italiani. Tali parole più che propriamente italiane sono meridionalismi; infatti coloro che partivano alla volta delle Americhe erano perlopiù dialettofoni che trapiantavano nell'inglese americano termini appartenenti alle varietà regionali del Mezzogiorno.

Al citato scenario si aggiungerà la marcata differenziazione degli italianismi culinari nell'inglese parlato negli Stati Uniti e in Inghilterra. Di conseguenza, iniziano a formarsi

${ }^{6}$ Cfr. DIFIT. 
italianismi enogastronomici propriamente dell'inglese americano come ad esempio fettunta (Haller, 1991).

Il fenomeno è vivissimo soprattutto nel Novecento, e particolarmente negli ultimi decenni, quando non si tratta più soltanto di un fatto legato alle migrazioni e alle tradizioni dei gruppi sociali, ma di una realtà che si inserisce nella nuova dimensione globale dell'era industriale e rende possibile la circolazione delle merci con ritmi e modalità prima impensabili. La larga reperibilità dei prodotti italiani anche più caratteristici, la creazione insomma di un mercato globale che induce la riconoscibilità del prodotto e del marchio (pensiamo alla caratura internazionale di alcuni dei nostri gruppi alimentari) comporta al tempo stesso la diffusione delle parole (Frosini, 2012: 104).

La globalizzazione e la facilità di incontro tra culture e lingue diverse ha solamente accelerato un processo ben radicato e presente fin dai secoli scorsi. Di conseguenza il fenomeno è in rapido mutamento e produce cambiamenti immediati nelle diverse lingue, soprattutto negli ambiti della gastronomia e dell'enologia nei quali l'Italia è modello di esportazione e di imitazione in tutto il mondo.

\section{COME RINTRACCIARE UN ITALIANISMO?}

Il fenomeno degli italianismi accomuna in misura più o meno intensa la maggior parte delle lingue con le quali l'italiano è venuto a contatto per ragioni storiche ed economiche. Se da un lato molti italianismi sono prestiti non adattati e presentano la stessa grafia dell'italiano, rendendo l'individuazione molto semplice, dall'altro molti lessemi hanno cambiato notevolmente la loro forma, adattandosi alla lingua di arrivo come accaduto per l'inglese artichoke. Il lessema deriva da sostantivo: articiocco ${ }^{7}$. Tuttavia, il termine si è adattato alla lingua inglese al punto da renderne irriconoscibile la provenienza. Pertanto, il vocabolo rispetta le regole e le strutture morfosintattiche appartenenti alla lingua inglese, come la regola di formazione del plurale (artichokes).

Inoltre, è necessario prestare attenzione agli pseudoitalianismi, formazioni che sembrano italiane ma non lo sono, per esempio la locuzione tutti frutti, che si trova in molte lingue nel senso di "frutta mista", poi "gelato misto", quindi usato per qualsiasi mixtum compositum ${ }^{8}$.

Lo strumento ideale per l'individualizzazione di un italianismo in una qualsiasi lingua è il vocabolario, che raccoglie i vocaboli usati da un numero sufficientemente ampio di parlanti per un ampio lasso temporale. Di conseguenza, un italianismo entra a far parte di un'altra lingua quando è ampiamente adoperato dai suoi parlanti e rintracciabile sul dizionario. Molti vocabolari, oltre a fornire il significato del lessema ricercato e i relativi sinonimi e contrari, forniscono indicazioni legate alla provenienza della parola, rendendo così più agevole la ricerca.

Un ulteriore strumento utile al riconoscimento degli italianismi è il già citato DIFIT, inserito all'interno del progetto OIM, Osservatorio degli italianismi nel mondo, di cui si tratterà nel paragrafo successivo.

7 Variante linguistica dell'Italia settentrionale per riferirsi al termine carciofo.: Cfr., per esempio, https://dizionaripiu.zanichelli.it/la-parola-e-servita/mille-modi-di-dire-carciofo/

8 Cfr. http://www.treccani.it/enciclopedia/italianismi_(Enciclopedia-dell\%27Italiano)/ 


\subsection{Osservatorio degli Italianismi nel Mondo}

L'Osservatorio degli Italianismi nel Mondo (OIM) mira alla costituzione di una banca dati che possa raccogliere tutte le parole italiane e di origine italiana entrate nell'uso di altre lingue. Inserito tra i progetti strategici dell'Accademia della Crusca all'interno del portale VIVIT $^{9}$, è pensato come punto di riferimento per la raccolta, verifica e condivisione di italianismi diffusi in diverse lingue del mondo. Nella banca dati è inserito il materiale raccolto per il DIFIT diretto da Harro Stammerjohann (già consultabile online all'indirizzo difit.italianismi.org) e per altri studi comunque in corso di elaborazione: rientra negli obiettivi del progetto l'allargamento immediato a altre tre importanti lingue neolatine (spagnolo, catalano e portoghese) alle quali vanno aggiunte ungherese e polacco, per cui esistono già ricerche avviate.

Il progetto è diretto da Matthias Heinz e Luca Serianni e si avvale di un gruppo di ricerca internazionale, composto da unità operanti presso le università di Barcellona, Budapest, Cracovia, Dresda, Firenze, Malta, Milano, Roma, Salisburgo, Siviglia, Toronto, Università della Calabria, Varsavia e altre ${ }^{10}$.

Ad oggi, l'Osservatorio degli Italianismi nel Mondo presenta più di $12.000^{11}$ italianismi registrati, numero già di per sé rilevante, ma in continuo aumento grazie all'integrazione dei risultati di nuove ricerche. Alle lingue già citate in precedenza sono state aggiunte infatti sia lingue europee come il maltese ed il russo, che lingue extraeuropee come il cinese e l'arabo.

L'OIM consente di ricavare informazioni di tipo storico-etimologico sulla voce italiana di partenza e di conoscere le caratteristiche principali per ogni lingua in cui il prestito è attestato. In particolare: forma grafica; genere e categoria grammaticale; data d'ingresso; eventuale uscita d'uso; ambito d'uso, attestazioni. Per ogni lingua sono offerti prospetti introduttivi che descrivono la storia linguistica di ciascuna varietà nella quale è censito l'apporto italiano; la descrizione lessicografica; il metodo per la raccolta degli italianismi; la valutazione d'insieme dei prestiti; le corrispondenze grafico-fonetiche ${ }^{12}$.

Le lingue non sono sistemi statici, esse mutano notevolmente con il passare del tempo $\mathrm{e}$ in base alle scelte dei parlanti, di conseguenza il progetto è in continua evoluzione ed aggiornamento sia per quanto riguarda le lingue già facenti parte del progetto sia per quelle in divenire di cui già esistono studi preliminari come è il caso del romeno, del ladino dolomitico o del finlandese ${ }^{13}$.

\subsection{Dizionario di italianismi in inglese, francese e tedesco}

Consultabile sia in versione cartacea che online, il DIFIT consente non solo di ricercare gli italianismi presenti in francese, tedesco e in inglese, ma anche di filtrare i lessemi interessati in base alla categoria grammaticale, alla prima attestazione, al settore o al campo semantico, al registro espressivo e in base anche al tipo di prestito.

$\mathrm{Nel}$ presente lavoro ci si è serviti del DIFIT soprattutto per gli italianismi legati all'ambito enogastronomico presenti in lingua inglese. Utilizzando, il dizionario come

\footnotetext{
9 «Archivio digitale integrato di materiali didattici, testi e documentazioni iconografiche e multimediali per la conoscenza all'estero del patrimonio linguistico e storico-culturale italiano, con particolare riguardo e destinazione a italiani all'estero di seconda e terza generazione» (http://difit.italianismi.org/).

${ }^{10}$ La presentazione del progetto è disponibile online: http://www.italianismi.org/home/progetto.

${ }^{11}$ Dati al 21/07/20.

12 Ivi.

${ }^{13}$ Cfr. Dimitrescu (2003), Videsott (2001), Pinomaa (2018).
} 
(C) Italiano LinguaDue 2. 2021. D. Della Pietra, Gli italianismi enogastronomici recenti nella lingua inglese: un'analisi sui corpora

banca dati per la verifica della presenza degli italianismi oggetto di studio nell'elaborato, ricavati dall'Oxford English Dictionary, nel DIFIT.

\section{GASTRONIMI ITALIANI IN INGLESE DI ORIGINE RECENTE: PRESENTAZIONE DEI LESSEMI RILEVATI, ACQUISIZIONE TERMINOLOGICA E METODOLOGIA DI ANALISI}

Gli italianismi oggetto di studio sono stati rintracciati all'interno dell'Oxford English Dictionary, grazie alla consultazione degli aggiornamenti su base trimestrale, disponibili online, che rendono noti i vocaboli messi a lemma dal dizionario inglese.

Sono stati accolti i forestierismi chiaramente di origine italiana identificati come prestiti non adattati (eccezion fatta per broccoli rabe, adattamento di broccolo rapa). Sono stati, invece, scartati i lessemi la cui reale origine non è ufficialmente attestata. È stato possibile stilare un elenco, qui di seguito riportato, di italianismi afferenti al mondo dell'enogastronomia messi a lemma dal dizionario inglese dal 2000 ad oggi.

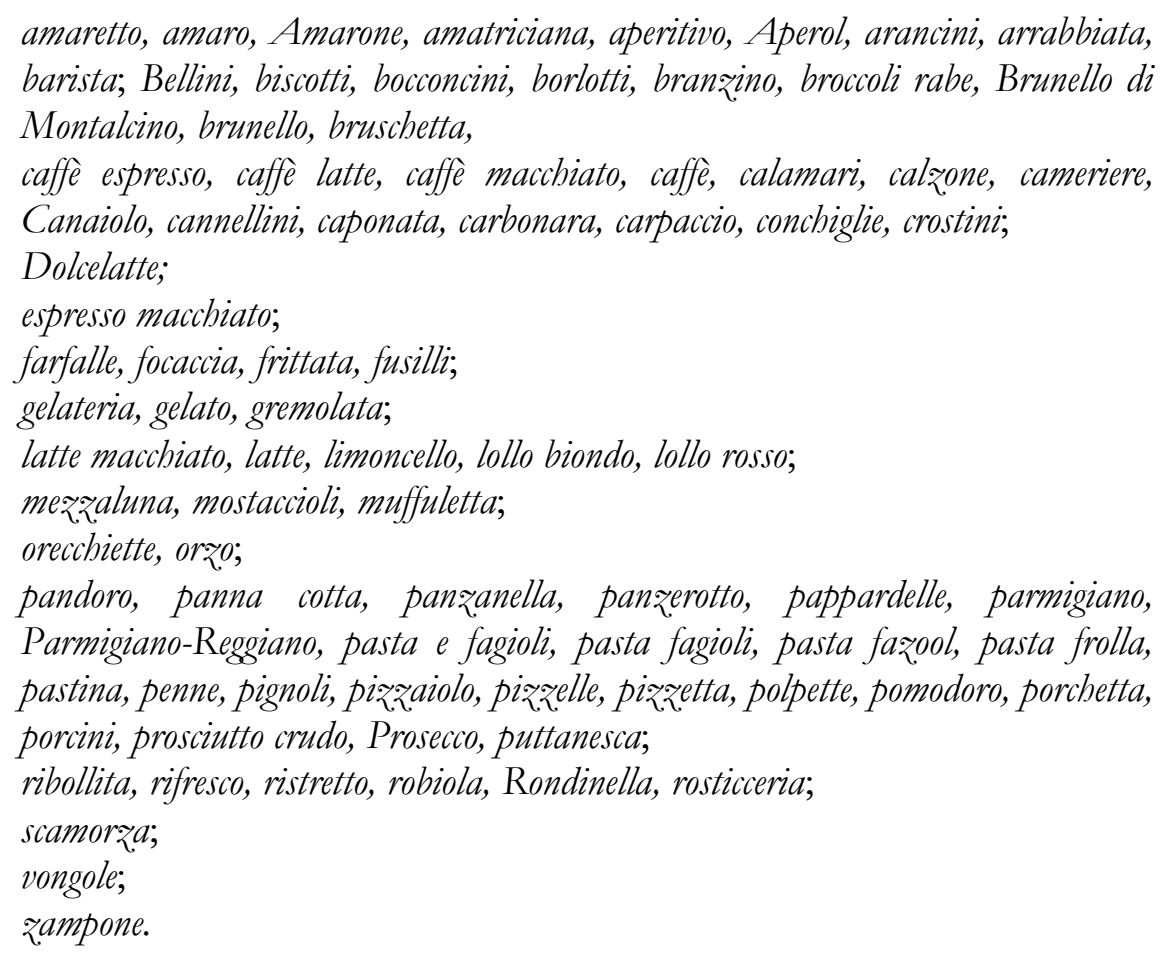

Viste le numerose aree semantiche appartenenti alla sfera dell'enogastronomia ai quali appartengono i lessemi rilevati, si è proceduto alla suddivisione dei medesimi in 21 classi semantiche: 1. caffè e polirematiche, 2. dolci, 3. figure professionali, 4. formaggi, 5. latte e polirematiche, 6. liquori, 7. modalità di preparazione e impasti, 8. nome del piatto, 9. nomi commerciali, 10. occasioni, 11. pane e piatti a base di pane, 12. pesce, 13. pietanze a base di carne, 14. pietanze a base di latte e/o uova, 15. punti vendita, 16. salsa o condimento, 17. salumi, 18. tipologie di pasta, 19. utensili da cucina, 20. verdure, legumi e semi, 21. vini e cocktail composti da vini.

La ripartizione dei lessemi in base alla classe semantica è stata raccolta nella seguente tabella ${ }^{14}$ :

${ }^{14}$ I lessemi contrassegnati con l'asterisco $\left(^{*}\right)$ presentano in italiano un doppio significato: considerata la prospettiva di analisi, è stato scelto di categorizzarle in base al significato usato nella lingua inglese. Nei casi 
(C) Italiano LinguaDue 2. 2021. D. Della Pietra, Gli italianismi enogastronomici recenti nella lingua inglese: un'analisi sui corpora

Tabella 2. Ripartizione dei lessemi in base alla classe semantica

\begin{tabular}{|c|c|c|}
\hline Classe semantica & Italianismi & Numero \\
\hline $\begin{array}{l}\text { Caffè e } \\
\text { polirematiche }\end{array}$ & $\begin{array}{l}\text { Caffè, Caffè espresso, Caffè latte, Caffè macchiato, } \\
\text { Espresso macchiato, Ristretto. }\end{array}$ & 6 \\
\hline Dolci & $\begin{array}{l}\text { Biscotti, Gelato, Mostaccioli*, Pandoro, Panna cotta, } \\
\text { Pizzelle* }\end{array}$ & 6 \\
\hline Figure professionali & Barista, Cameriere, Pizzaiolo & 3 \\
\hline Formaggi & Parmigiano, Parmigiano-Reggiano, Robiola, Scamorza & 4 \\
\hline $\begin{array}{l}\text { Latte e } \\
\text { polirematiche }\end{array}$ & Latte, Latte macchiato & 2 \\
\hline Liquori & Amaretto*, Amaro, Aperitivo, Limoncello & 4 \\
\hline $\begin{array}{l}\text { Modalità di } \\
\text { preparazione e } \\
\text { impasti }\end{array}$ & Carpaccio, Pasta frolla & 2 \\
\hline Nome del piatto & $\begin{array}{l}\text { Arancini, Calzone, Caponata, Pasta e fagioli, Pasta fagioli, } \\
\text { Pasta fazool, Ribollita }\end{array}$ & 7 \\
\hline Nomi commerciali & Aperol, Dolcelatte & 2 \\
\hline Occasioni & Rinfresco & 1 \\
\hline $\begin{array}{l}\text { Pane e piatti a base } \\
\text { di pane }\end{array}$ & $\begin{array}{l}\text { Bruschetta, Crostini, Focaccia, Muffuletta, Panzanella, } \\
\text { Panzerotto, Pizzetta }\end{array}$ & 7 \\
\hline Pesce & Branzino, Calamari, Vongole & 3 \\
\hline $\begin{array}{l}\text { Pietanze a base di } \\
\text { carne }\end{array}$ & Polpette, Porchetta & 2 \\
\hline $\begin{array}{l}\text { Pietanze a base di } \\
\text { latte e/o uova }\end{array}$ & Bocconcini*, Frittata & 2 \\
\hline Punti vendita & Gelateria, Rosticceria & 2 \\
\hline Salsa o condimento & Amatriciana, Arrabbiata, Carbonara, Gremolata, Puttanesca & 5 \\
\hline Salumi & Prosciutto crudo, Zampone & 2 \\
\hline Tipologie di pasta & $\begin{array}{l}\text { Conchiglie, Farfalle, Fusilli, Orecchiette, Orzo, Pappardelle, } \\
\text { Pastina, Penne }\end{array}$ & 8 \\
\hline Utensili da cucina & Mezzaluna & 1 \\
\hline $\begin{array}{l}\text { Verdure, legumi e } \\
\text { semi }\end{array}$ & $\begin{array}{l}\text { Borlotti, Broccoli rabe, Cannellini, Lollo biondo, Lollo } \\
\text { rosso, Pignoli, Pomodoro, Porcini }\end{array}$ & 8 \\
\hline $\begin{array}{l}\text { Vini e cocktail } \\
\text { composti da vini }\end{array}$ & $\begin{array}{l}\text { Amarone, Bellini, Brunello, Brunello di Montalcino, } \\
\text { Canaiolo, Prosecco, Rondinella }\end{array}$ & 7 \\
\hline
\end{tabular}

in cui sia attestato il doppio significato anche in lingua inglese, il lessema è stato inserito nella categoria appartenente al significato più frequente. 
(C) Italiano LinguaDue 2. 2021. D. Della Pietra, Gli italianismi enogastronomici recenti nella lingua inglese: un'analisi sui corpora

\section{CONSIDERAZIONI LINGUISTICHE}

Gli influssi linguistici della lingua italiana sulla lingua inglese, specie per quanto riguarda l'ambito enogastronomico, sono tangibili e costanti.

Negli ultimi vent'anni, anche grazie al processo di globalizzazione e alla diffusione capillare di internet, i contatti fra le due lingue si sono acuiti notevolmente e di conseguenza sono entrati a far parte della lingua inglese gastronimi di origine italiana riguardanti non solamente pietanze che caratterizzano la cultura italiana, ma anche gli ingredienti e gli utensili utili per la loro preparazione.

Buona parte dei gastronimi presi in esame sono considerati prestiti di lusso, cioè importati direttamente da lingue che dominano l'ambito di interesse e che godono di un prestigio riconosciuto anche su altre lingue. Tale influenza comporta che spesso i nuovi lessemi introdotti non subiscono sostanziali adattamenti nella lingua di arrivo e mantengono la forma originale. Tuttavia, non è raro che i parlanti adattino i lessemi importati sulla base della grammatica e delle regole morfosintattiche della propria lingua.

Nei prossimi paragrafi sono riportate alcune riflessioni sui lessemi oggetto di studio legate ai diversi livelli linguistici, eccezion fatta per il livello fonetico perché, come nota Pinnavaia (2006: 14), la pronuncia inglese dei prestiti enogastronomici «rimane essenzialmente inalterata, o meglio è adattata in modo da rimanere il più vicino possibile all'originale».

\subsection{Considerazioni quantitative}

Da un punto di vista quantitativo è possibile notare come 58 lessemi su 84, ovvero il $69 \%$, non sia inserito nel DIFIT, come dimostrato dal seguente grafico:

Grafico 1. Presenza dei termini nel DIFIT

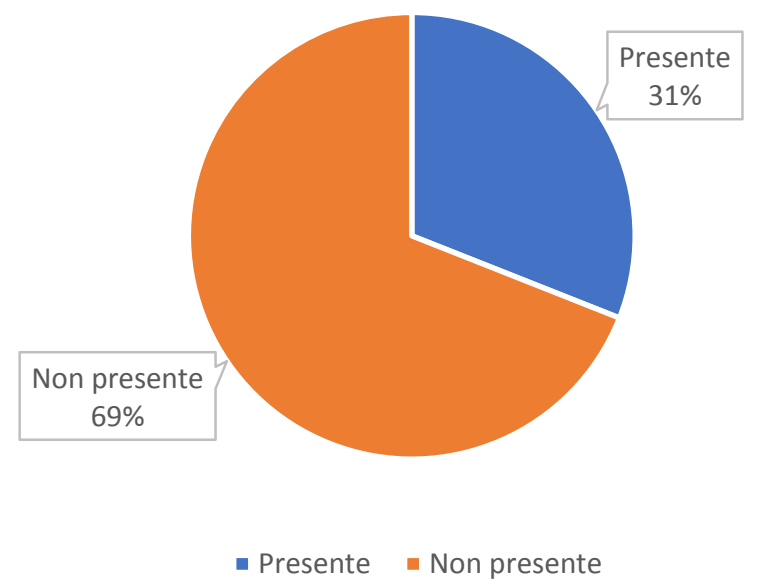

Segue l'elenco dei prestiti non presenti nel DIFIT:

amaro, amarone, amatriciana, aperitivo, aperol, arancini, arrabbiata;

barista, bellini, biscotto, bocconcini, borlotti, branzino, broccoli rabe, Brunello di Montalcino, brunello;

caffe macchiato, cameriere, canaiolo, cannellini, caponata, carbonara;

espresso macchiato; 
(C) Italiano LinguaDue 2. 2021. D. Della Pietra, Gli italianismi enogastronomici recenti nella lingua inglese: un'analisi sui corpora

gelato, gremolata;

limoncello, lollo biondo, lollo rosso;

mezzaluna, mostaccioli, muffuletta;

orecchiette;

pandoro, panna cotta, panzerotto, parmigiano-reggiano, pasta e fagioli, pasta fagioli, pasta fazool, pasta frolla, pastina, pizzaiolo, pizzelle, pizzetta, polpetta, pomodoro, porchetta, porcino, puttanesca;

ribollita, rinfresco, ristretto, robiola, rondinella, rosticceria;

scamorza;

vongole;

zampone.

Per quanto concerne il numero di occorrenze, si evidenzia una certa proporzionalità della presenza terminologica nei corpora analizzati, legata alla grandezza di ogni singolo corpus. Infatti, il New York Times Cooking (NYTC) registra 1.662 ricette di piatti italiani su 13.954 ricette totali, mentre il BBC Good Food (BBCGF) 969 su 9.609.

Grafico 2. Ricette di piatti italiani registrati dal NYTC e dal BBCGF

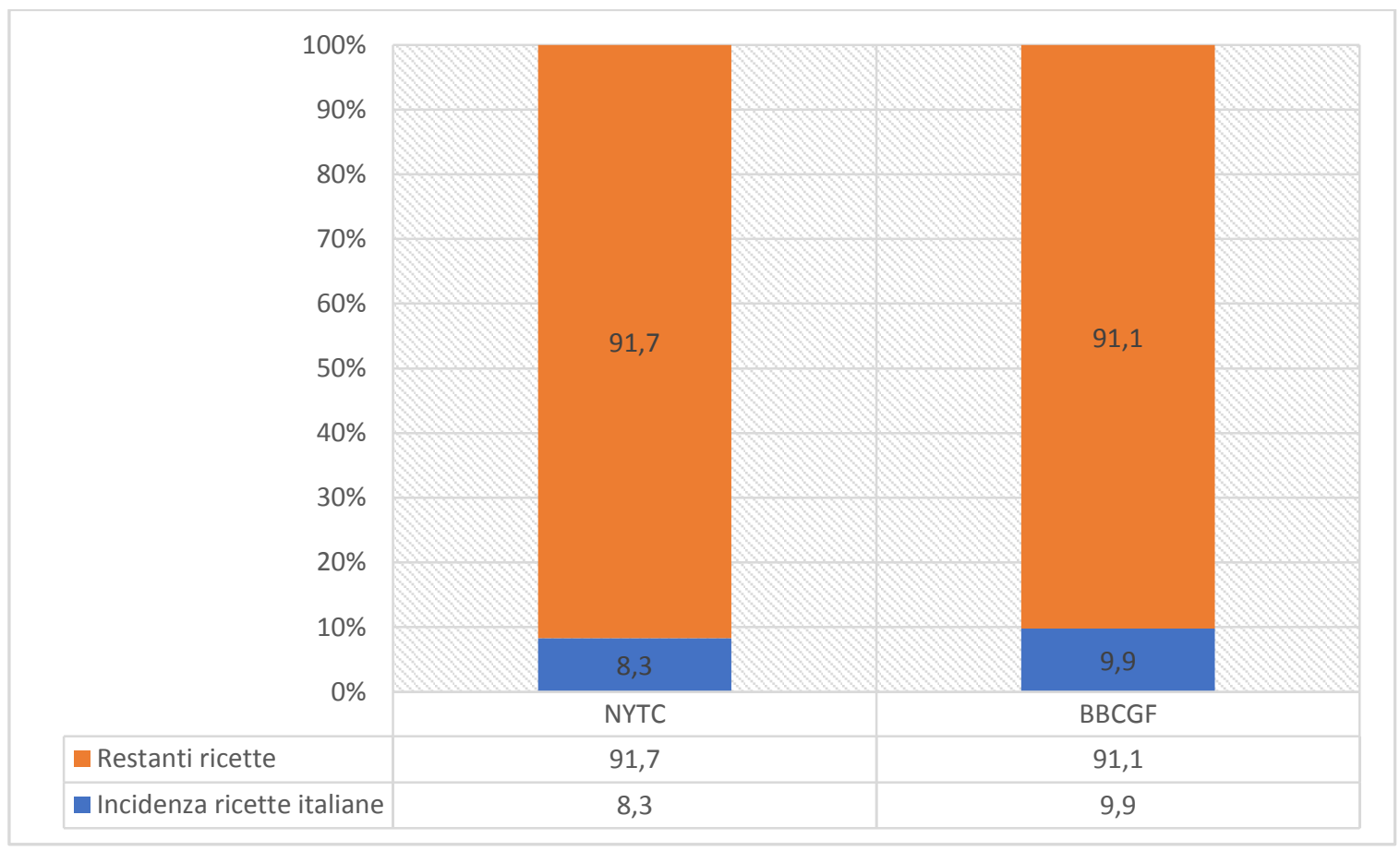

Tuttavia, osservando l'incidenza delle ricette italiane e di conseguenza anche degli italianismi per quanto concerne ambedue i corpora, è possibile notale come essa sia maggiore nel BBCGF (circa il 10\% sulle ricette totali) rispetto al quotidiano statunitense dove le ricette italiane rappresentano ben l' $8.3 \%$ delle ricette totali.

\subsubsection{Confronto tra i corpora}

Prendendo in esame gli italianismi oggetto di studio nei tre corpora linguistici è stato possibile compilare la seguente tabella nella quale si registra il numero di occorrenze di ogni lessema. 
(C) Italiano LinguaDue 2. 2021. D. Della Pietra, Gli italianismi enogastronomici recenti nella lingua inglese: un'analisi sui corpora

Tabella 3. Numero di occorrenze dei lessemi

\begin{tabular}{|c|c|c|c|}
\hline & EnTenTen15 & NYTC & BBCGF \\
\hline Amaretto & 1.024 & 46 & 56 \\
\hline Amaro & 2.895 & 34 & 1 \\
\hline Amarone & 831 & - & - \\
\hline Amatriciana & 155 & 6 & 5 \\
\hline Aperitivo & 930 & 28 & 5 \\
\hline Aperol & 401 & 49 & 14 \\
\hline Arancini & 358 & 13 & 8 \\
\hline Arrabbiata & 96 & 4 & 8 \\
\hline Barista & 11.126 & 1 & 5 \\
\hline Bellini & 4.394 & 2 & 6 \\
\hline Biscotto & 38 & 37 & 13 \\
\hline Bocconcini & 110 & 4 & - \\
\hline Borlotti & 203 & 24 & 7 \\
\hline Branzino & 157 & 6 & - \\
\hline Broccoli rabe & 518 & 85 & - \\
\hline Brunello & 1.196 & - & - \\
\hline Brunello di Montalcino & 261 & 2 & 1 \\
\hline Bruschetta & 1.951 & 32 & 49 \\
\hline Caffè & 1.303 & 1 & 2 \\
\hline Caffè Espresso & 8 & - & - \\
\hline Caffè latte & 79 & 3 & 13 \\
\hline Caffè macchiato & 12 & - & 1 \\
\hline Calamari & 3.483 & 69 & 10 \\
\hline Calzone & 1.855 & 228 & 12 \\
\hline Cameriere & 29 & - & - \\
\hline Canaiolo & 61 & - & - \\
\hline Cannellini & 590 & 109 & 68 \\
\hline Caponata & 306 & 11 & 10 \\
\hline Carbonara & 1.440 & 17 & 75 \\
\hline Carpaccio & 1.050 & 16 & 10 \\
\hline Conchiglie & 47 & 1 & 1 \\
\hline Crostini & 1.230 & 188 & 26 \\
\hline Dolcelatte & 15 & - & 7 \\
\hline
\end{tabular}


(C) Italiano LinguaDue 2. 2021. D. Della Pietra, Gli italianismi enogastronomici recenti nella lingua inglese: un'analisi sui corpora

\begin{tabular}{|c|c|c|c|}
\hline & EnTenTen15 & NYTC & BBCGF \\
\hline Espresso macchiato & 29 & - & - \\
\hline Farfalle & 250 & 40 & 8 \\
\hline Focaccia & 2.264 & 51 & 27 \\
\hline Frittata & 2.124 & 92 & 71 \\
\hline Fusilli & 479 & 43 & 23 \\
\hline Gelateria & 600 & - & 1 \\
\hline Gelato & 8.180 & 15 & 6 \\
\hline Gremolata & 238 & 31 & 21 \\
\hline Latte & 16.547 & 2 & 9 \\
\hline Latte macchiato & 136 & - & - \\
\hline Limoncello & 1.030 & 3 & 18 \\
\hline Lollo biondo & 2 & - & - \\
\hline Lollo rosso & 40 & 1 & 1 \\
\hline Mezzaluna & 185 & - & - \\
\hline Mostaccioli & 98 & - & - \\
\hline Muffuletta & 533 & 1 & 1 \\
\hline Orecchiette & 263 & 36 & 7 \\
\hline Orzo & 982 & 72 & 35 \\
\hline Pandoro & 96 & - & 4 \\
\hline Panna cotta & 1.597 & 17 & 25 \\
\hline Panzanella & 315 & 32 & 15 \\
\hline Panzerotto & 23 & - & - \\
\hline Pappardelle & 427 & 34 & 21 \\
\hline Parmigiano & 1.124 & 1 & - \\
\hline Parmigiano-Reggiano & 627 & 228 & 3 \\
\hline Pasta e fagioli & 78 & 3 & 1 \\
\hline Pasta fagioli & 65 & 2 & 1 \\
\hline Pasta fazool & 15 & - & - \\
\hline Pasta frolla & 36 & - & 2 \\
\hline Pastina & 70 & 3 & - \\
\hline Penne & 2.762 & 114 & 58 \\
\hline Pizzaiolo & 239 & 2 & - \\
\hline Pizzelle & 256 & 4 & - \\
\hline Pizzetta & 49 & 16 & 5 \\
\hline Polpetta & 15 & - & 2 \\
\hline
\end{tabular}


(C) Italiano LinguaDue 2. 2021. D. Della Pietra, Gli italianismi enogastronomici recenti nella lingua inglese: un'analisi sui corpora

\begin{tabular}{|l|c|c|c|}
\hline & EnTenTen15 & NYTC & BBCGF \\
\hline Pomodoro & 1.593 & 7 & 9 \\
\hline Porchetta & 481 & 8 & 7 \\
\hline Porcino & 118 & 143 & - \\
\hline Prosciutto crudo & 62 & - & 50 \\
\hline Prosecco & 3.425 & 13 & 16 \\
\hline Puttanesca & 279 & 15 & - \\
\hline Ribollita & 157 & 9 & - \\
\hline Rinfresco & 6 & - & - \\
\hline Ristretto & 29 & 1 & - \\
\hline Robiola & 88 & 2 & - \\
\hline Rondinella & 216 & - & - \\
\hline Rosticceria & 30 & - & 5 \\
\hline Scamorza & 110 & - & - \\
\hline Vongole & 154 & 4 & - \\
\hline Zampone & 44 & & - \\
\hline
\end{tabular}

Analizzando il numero di occorrenze è possibile notare come i lessemi compaiano più spesso nel corpus EnTenTen15, vista la grandezza di quest'ultimo rispetto agli altri due. Tuttavia, vi sono alcuni dati numerici da prendere in considerazione. Nel corpus appena citato gli italianismi che registrano più occorrenze e un numero superiore alle 3.000 sono: latte, barista, gelato, Bellini, calamari e prosecco; i quali tuttavia non hanno una presenza così ricorrente nei corpora dei due enti di informazione. Ad eccezione dell'italianismo calamari il quale presenta ben 69 occorrenze per quanto concerne il NYTC e 10 nel BBCGF.

Analogamente a calamari, vi sono alcuni lessemi che, in proporzione, risultano più presenti negli altri corpora: è il caso di porcino, biscotto oppure cannellini.

Osservando gli italianismi che occorrono di più nel NYTC si evince come i termini più ricorrenti siano: calzone (228), Parmigiano-Reggiano (228), crostini (188), porcino (143), penne (144) e cannellini (109). Per quanto riguarda il corpus ricavato da BBCGF il termine che registra più presenze è carbonara (75), seguito da frittata (71), cannellini (68) e penne (58).

Mettendo a confronto i risultati delle occorrenze dei corpora dei due enti di informazione è possibile constatare come il numero delle occorrenze nel NYTC sia numericamente superiore al BBCGF. Eppure, in alcuni italianismi tale tendenza non sussiste come accade con carbonara che registra 75 occorrenze contro le 17 del New York Times Cooking, amaretto (56-46) o Prosecco che appare 50 volte nel corpus britannico rispetto alle 13 del NYTC. Quest'ultimo dato è giustificato dalla condizione che il lessema vanta nell'inglese britannico, ovvero di proprietary name come testimoniato anche dall'Oxford English Dictionary. 
(C) Italiano LinguaDue 2. 2021. D. Della Pietra, Gli italianismi enogastronomici recenti nella lingua inglese: un'analisi sui corpora

\subsection{Considerazioni morfosintattiche}

Da un punto di vista morfosintattico si riscontra in alcuni lessemi il travisamento del numero del lessema, nei casi in cui il termine entra nella lingua inglese nella sua forma plurale, esso molte volte viene decodificato come una marca di singolare. Di conseguenza i parlanti tendono a "ripluralizzare" il termine, seguendo le norme morfosintattiche della lingua inglese, giustapponendo ad esempio il suffisso indigeno $-s$, come notato anche da Perissinotto (2015: 277). Avremo quindi formazioni del tipo: biscotti-biscottis oppure calamari-calamaries.

Vi è, tuttavia, un cospicuo numero di lessemi; (amaro, aperitivo, calzone, focaccia, gelateria, gelato, pizzaiolo, pizzetta, polpetta e rosticceria) che vede la presenza sia della marca di plurale ricavata dalla lingua italiana, sia di quella adattata. Ad esempio, nei corpora di controllo rosticceria si trova sia nella forma rosticcerie sia rosticcerias.

\subsection{Considerazioni ortografiche}

Da un punto di vista ortografico sono due le dinamiche che caratterizzano i prestiti circolanti nella lingua inglese: l'alternanza tra maiuscola e minuscola e l'incertezza nelle doppie. Molto spesso in alcuni lessemi, per esempio amarone e bellini, è possibile notare la sovrapposizione fra nome comune e nome di marchio, come già notato da Pinnavaia (2006: 15). Inoltre, spesso le consonanti geminate non vengono rese in inglese, come accade nei nessi $b b$ o $t t$, presenti nei sostantivi arrabbiata e puttanesca. Tale dinamica è legata, come spiega ancora Pinnavaia (ivi: 14), alla difficile percezione di tali nessi all'orecchio anglofono.

\subsection{Considerazioni lessicali}

Per quanto concerne il livello lessicale è possibile notare che ad alcuni termini spesso vengano giustapposti elementi lessicali appartenenti al sistema linguistico di arrivo, atti a chiarire il significato del forestierismo. Per bisogno di chiarezza, tuttavia, al termine alloglotto viene talvolta aggiunto un elemento lessicale indigeno a formare quello che Iamartino (2001: 61) definisce "composto chiarificante", che specifica il significato o il campo semantico di appartenenza del prestito (ballerina shoe, ponente wind, romano cheese), fino a duplicare mediante un traducente il significato del prestito italiano creando così una vera e propria tautologia (prosciutto ham). È il caso dei lessemi muffuletta, amatriciana, pomodoro e pignoli, i quali in alcuni casi divengono, come attestato dall'OED, muffuletta sandwrich, amatriciana sauce, pomodoro sauce e pignoli nut. Inoltre, è possibile notare come l'Oxford English Dictionary metta a lemma in alcuni casi sia lessemi quali brunello e parmigiano sia la loro forma polirematica: Brunello di Montalcino o Parmigiano Reggiano.

\subsection{Considerazioni semantiche}

A livello semantico, per quanto riguarda alcuni lessemi, è possibile notare tre fenomeni, ovvero: 
(C) Italiano LinguaDue 2. 2021. D. Della Pietra, Gli italianismi enogastronomici recenti nella lingua inglese: un'analisi sui corpora

a) risemantizzazioni, come accade con i lessemi latte, pizzelle, mostaccioli e orzo.

Latte, come nota anche Pinnavaia (2006: 16) non è l'equivalente di milk, ma è usato per riferirsi al prestito presente in lingua inglese caffe latte, di cui latte è la forma abbreviata.

Il lessema pizzella nella lingua italiana denota una tipologia di pizza, tuttavia nella lingua inglese, soprattutto per la variante americana, il lessema designa un tipo di biscotto consumato nelle occasioni festive.

Per quanto riguarda mostaccioli, nella lingua italiana il lessema denota un dolce natalizio, mentre nella lingua inglese questa parola è usata per fare riferimento a un particolare formato di pasta.

Anche il termine oryo vede una sostanziale modifica semantica: mentre nella lingua italiana orzo denota un cereale, nella lingua inglese viene utilizzato per fare riferimento a un formato di pasta.

b) restringimenti di significato, come nel caso di biscotto. Il termine nella lingua inglese è utilizzato per fare riferimento ad una sorta di biscotto da tè, sottile e allungato.

c) ampliamenti del significato, come accade con il lessema vongole che oltre a designare come nella lingua di origine il mollusco, in inglese designa anche il condimento ricavato dal mollusco stesso.

\subsection{Considerarioni sociolinguistiche diatopiche}

Da un punto di vista sociolinguistico è possibile evincere che tra gli 84 lessemi fin qui presi in esame, 27 presentano una tipica provenienza geografica (v. Tabella 3), ovvero il $32 \%$ rispetto agli italianismi rilevati. Tale classificazione è stata ricavata grazie al confronto dei dati ricavati dal Grande dirionario dell'uso di De Mauro (GDU), dal Dizionario etimologico dei dialetti italiani di Cortelazzo e Marcato (DEDI) e del Diæionario delle cucine regionali italiane di Paola Gho (DCRI), i quali permettono di analizzare la rilevanza regionale sugli italianismi oggetto di studio.

Tabella 3. Provenienza geografica degli italianismi $i^{15}$

\begin{tabular}{|l|l|}
\hline \multicolumn{1}{|c|}{ Regione di provenienza } & \multicolumn{1}{c|}{ Italianismo } \\
\hline Abruzzo & Pizzelle*, \\
\hline Basilicata & Orecchiette* \\
\hline Campania & Mostoccioli, limoncello, pasta fazool, puttanesca \\
\hline Emilia-Romagna & Parmigiano-Reggiano, parmigiano, zampone \\
\hline Lazio & Porchetta, bocconcini, amatriciana, arrabbiata, carbonara \\
\hline Liguria & Panna cotta \\
\hline Lombardia & Gremolata \\
\hline Molise & Pizzelle* \\
\hline Puglia & Panzerotto, orecchiette* \\
\hline
\end{tabular}

${ }^{15}$ Nella tabella 3 alcuni lessemi sono stati contrassegnati $\left(^{*}\right)$ in virtù della loro origine regionale dibattuta fra due o più regioni. Tali lessemi, di conseguenza verranno ripetuti. 
(C) Italiano LinguaDue 2. 2021. D. Della Pietra, Gli italianismi enogastronomici recenti nella lingua inglese: un'analisi sui corpora

\begin{tabular}{|l|l|}
\hline Sicilia & Arancini, caponata, muffuletta \\
\hline Toscana & Ribollita, brunello, Brunello di Montalcino \\
\hline Veneto & Pandoro, carpaccio, branzino, amarone, rondinella \\
\hline
\end{tabular}

I risultati ottenuti evidenziano una presenza consistente di italianismi originari soprattutto da regioni quali: Lazio, Veneto, Campania, Sicilia, Emilia-Romagna e Toscana.

Di seguito è possibile osservare la rappresentazione grafica dei dati sopracitati.

Grafico 3. Italianismi su base regionale

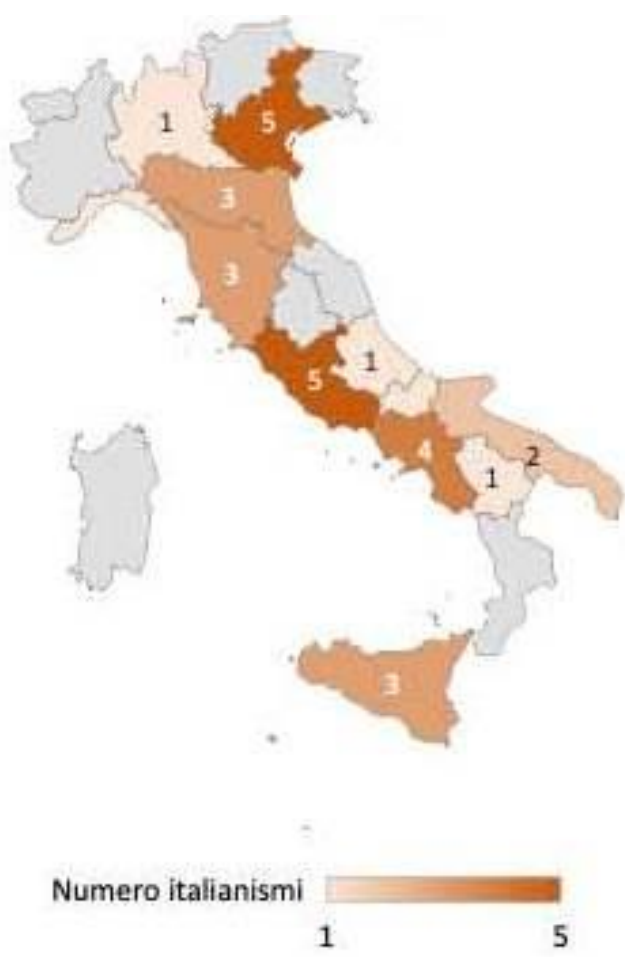

Fra i 27 lessemi sopraelencati 3 sono da considerare come regionalismi o dialettismi: branzino, pizzelle e gremolata.

Il lessema branzino è un regionalismo veneto, come indicato anche dal GDU, che denota il pesce identificato come spigola nellitaliano standard. Per pizzella si intende un dolce tipico dell'Italia centromeridionale conosciuto a livello nazionale come ferratella. Infine, la gremolata (adattamento di cremolata) è un condimento utilizzato per insaporire un prodotto tipico lombardo: l'osso buco.

Meritano un discorso a parte i lessemi pasta fazool e muffuletta. Entrambi di origine dialettale, rispettivamente napoletana e siciliana, sono di circolazione prettamente circoscritta agli Stati Uniti. Pasta fazool è un costrutto informale utilizzato nell'inglese americano derivante dal napoletano pasta e fasule per fare riferimento alla pasta e fagioli. Il lessema muffuletta, invece, deriva da muffulettu, lessema che nel dialetto siciliano indica una frittella di pasta di pane preparata tradizionalmente per la festa dell'Immacolata (Cortelazzo, Marcato, 2017: 290). Tuttavia, nell'inglese americano il lessema ha una referenzialità difforme dalle origini, infatti il sostantivo muffuletta è utilizzato per indicare un panino tipico dell'area di New Orleans come indicato anche dall'Oxford English Dictionary. 
La muffuletta ${ }^{16}$, secondo l'American Heritage Dictionary (AHD), è stata creata all'inizio del XX secolo da Salvatore Lupo, proprietario del Central Grocery, un negozio di alimentari tipicamente italiano situato nella città di New Orleans, il quale continua a vendere le famose muffulette tutt'oggi. Agli inizi del secolo scorso, New Orleans vantava una notevole presenza di braccianti agricoli di origine siciliana, i quali, spesso, tornando dai campi, andavano a pranzare nel negozio di alimentari del signor Lupo. I contadini, però, consumavano il loro pasto stando seduti in cima a delle casse o a dei barili, restando così in precario equilibrio e avendo non poche difficoltà nel mangiare. Di conseguenza, il proprietario dell'alimentari pensò che sarebbe stato più agevole per loro mangiare gli affettati e i formaggi all'interno di un comodo panino invece di mantenere in equilibrio il proprio piatto sulle gambe. Nasce così la muffuletta, un panino realizzato con l'omonimo pane tipico siciliano che nell'inglese americano prende il nome di muffuletta sandwich ed è simbolo della città di New Orleans.

\section{CONCLUSIONI}

Il comparto degli italianismi è un settore in continua evoluzione e riguarda sempre più lingue, di conseguenza progetti quali il DIFIT o l'OIM rivestono un ruolo di primo ordine nella catalogazione e documentazione dei lessemi di origine italiana presenti in lingue altre. I rapidi mutamenti che interessano tutti i sistemi linguistici impongono, di conseguenza, un costante impegno nell'aggiornamento di tali iniziative che misurano in maniera tangibile la presenza dell'italiano nelle lingue con cui esso viene a contatto ed è in questo scenario che si inserisce il presente lavoro.

Gli 84 lessemi rintracciati afferiscono a una molteplicità di ambiti del mondo enogastronomico. Dalle pietanze tipiche della cucina italiana, agli ingredienti utili alla preparazione del prodotto, passando per gli utensili. Sono stati rintracciati inoltre lessemi che fanno riferimento al mondo enogastronomico da un punto di vista indiretto come le figure lavorative ed i punti vendita.

Analizzando da un punto di vista quantitativo i lemmi oggetto di studio è interessante notare come un cospicuo numero di occorrenze che ogni italianismo fa registrare sia relativo non solo al corpus enTenTen15, ma anche alle sezioni enogastronomiche del New York Times e della BBC, a dimostrazione del fatto che gli italianismi oggetto di studio circolino con una certa fluidità non solo nell'inglese britannico, ma anche e soprattutto in quello americano.

Tenendo in considerazione il numero di occorrenze si evidenzia una certa proporzionalità della presenza terminologica nei corpora analizzati legata alla grandezza di ogni singolo corpus, infatti, l'enTenTen 15 comprende 13 miliardi di lessemi mentre il New York Times Cooking ed il BBC Good Food comprendono rispettivamente 13.954 e 9.609 ricette totali. Inoltre, è interessante notare l'incidenza delle ricette italiane paragonate a quelle totali presenti sul NYTC (ben 1.662 su 13.954 ovvero l'8\%) e sul BBCGF (969 su 9.609 complessive, precisamente il 10\%), numeri alquanto importanti se si pensa al numero infinito di culture gastronomiche esistenti.

Gli "italianismi recenti" analizzati non risultano come prestiti adattati, eccezion fatta per broccoli rabe derivato dall'italiano broccolo rapa.

Un elemento che accomuna gli 84 lessemi è la volontà da parte dei parlanti angloamericani di preservare la forma lessicale originale. Laddove vi siano discrepanze fra la forma originale e quella di arrivo esse sono ampiamente prevedibili come, ad esempio,

16 Per ulteriori informazioni consultare: https://ahdictionary.com/word/search.html?q=muffuletta. 
quelle di natura ortografica e morfosintattica. Un esempio è l'incertezza per quanto riguarda le consonanti geminate $b b$ e $t t$ in arrabbiata e puttanesca oppure il travisamento del numero del lessema, che interessa lessemi quali, ad esempio, biscotti oppure calamari. Lessemi che, seguendo le norme morfosintattiche della lingua inglese, vedono la giustapposizione del suffisso indigeno $-s$ al lessema originale, dando vita così ad una sorta di "doppio plurale".

Nei casi in cui l'italianismo non venga recepito appieno dai parlanti, si ricorre ai "composti chiarificanti", che specificano il significato o il campo semantico di appartenenza del prestito, rendendo così più accessibile il forestierismo. Come accade con i lessemi, amatriciana e pomodoro ai quali spesso viene giustapposto il sostantivo sauce, come evidenziato anche dall'OED.

Non tutti gli italianismi analizzati mantengono in toto il loro significato originale. Alcuni lessemi, come ad esempio latte oppure oræo presentano profonde risemantizzazioni, cambiamenti di significato radicali. D'altra parte, alcuni termini vedono un restringimento rispetto al significato originale come nel caso di biscotto oppure ampliamenti del significato lessicale come accade con il lessema vongole.

Infine, è interessante constatare come negli ultimi vent'anni siano entrati nella lingua inglese non solo termini appartenenti all'italiano standard, ma anche dialettismi, i quali hanno più fortuna in termini di circolazione nella lingua di approdo piuttosto che in quella di origine, quali pizzelle. Degni di nota sono anche i dialettismi ed i regionalismi come muffuletta e pasta fazool che hanno ampia circolazione nell'inglese americano a differenza dell'italiano standard.

Nell'articolo si è voluto analizzare gli italianismi legati alla sfera dell'enogastronomia, dimostrando come il settore - da tempi remoti fino a tempi recenti - ne sia uno dei principali erogatori.

\section{RIFERIMENTI BIBLIOGRAFICI}

Ayala Simón E. (2002), "Italianismos en DRAE 1992 (versión electrónica), heterogeneidad en las marcas del sector de las comidas" in Atti del XX Convegno dell'Associazione Ispanisti Italiani, Andrea Lippolis editore, Firenze, 37-50: https://cvc.cervantes.es/literatura/aispi/pdf/16/16_035.pdf.

Coluccia R. (2017), "Episodi e forme di diffusione della lingua italiana nel mondo (con qualche consioderazione sull'italiano in patria)", in Papa E., Cacia D. (a cura di), Di nomi e di parole. Studi in onore di Alda Rossebastiano, Società Editrice Romana, Roma, pp. 475-502:

https://www.academia.edu/35549556/Italiano_nel_mondo_e_italiano_in_patria_

Dimitrescu F. (2003), "Elemente italienești recente în limba română”, in Ead, Drumul neîntrerupt al limbii române, Editura Clusium, Cluj-Napoca, pp. 127-146.

Endruschat A. (2010), "Italianismi nel portoghese - lusismi nell'italiano", in Radatz H., Schlösser R. (a cura di), Donum Grammaticorum, Max Niemeyer Verlag, Berlino, pp. 29-64.

Frosini G. (2012), "La cucina degli italiani: tradizione e lingua dall'Italia al mondo", in Mattarucco G., Biffi M. (a cura di), Italiano per il mondo. Banca, commerci, cultura, arti, tradizioni, Accademia della Crusca, Firenze, pp. 85-107.

Haller H. W. (1991), "L'italianismo recente come riflesso dell'immagine italiana nella vita americana", in Coveri L. (a cura di), L'italiano allo specchio. Aspetti dell'italianismo recente, 
(C) Italiano LinguaDue 2. 2021. D. Della Pietra, Gli italianismi enogastronomici recenti nella lingua inglese: un'analisi sui corpora

Atti del Primo Convegno della Società Internazionale di linguistica e Filologia Italiana (Siena, 28- 31 marzo 1989), Vol. $2^{\circ}$, Rosenberg \& Sellier, Torino, pp. 1524.

Haller H. W. (2005), "L'italiano nei nomi dei ristoranti di New York", in Lid'O - Lingua italiana d'oggi, 2, pp. 331-352.

Iamartino G. (2001), "La contrastività italiano-inglese in prospettiva storica", in Rassegna italiana di linguistica applicata, XXXIII, 2-3, pp. 1-124.

Motolese M. (2012), Italiano lingua delle arti. Un'avventura europea (1250-1650), il Mulino, Bologna.

Ozbot M. (2008), "Alcuni cenni sugli italianismi in sloveno”, in Linguistica, 48, 1, pp. 159166:

https://www.dlib.si/stream/URN:NBN:SI:DOC-DXECEDPW/df7131aa-a7b147b8-a0db-91489ea18aee/PDF.

Perissinotto F. (2015). "Meal in Italy. Italianismi nel linguaggio gastronomico inglese e angloamericano", in Italiano LinguaDue, 7, 1, pp. 265-295:

https://riviste.unimi.it/index.php/promoitals/article/view/5022.

Pinnavaia L. (2006), "Il sapore delle parole: la terminologia inglese di origine italiana del cibo", in Colombo M., Iamartino G., Vidoni M. S. et. al. (a cura di), Mots palabras Words, LED - Edizioni Universitarie di Lettere Economia Diritto, Milano, pp. 7 23.

Pinomaa O. (2018), Gli italianismi nella lingua speciale della gastronomia: uno studio su libri di cucina pubblicati in finlandese tra il 1997 e il 2016. Tesi di laura magistrale, Istituto di Linguistica e traduzione, Facoltà di Lettere e Filosofia. università di Turku:

https://www.utupub.fi/bitstream/handle/10024/146522/Pinomaa_Outi_opinna yte.pde?sequence $=1$ \&isAllowed $=y$.

Rossi L. (2009), “Assaggi da un dizionario di italianismi nel mondo”, in Lingua Italiana, Treccani: https://www.treccani.it/magazine/lingua_italiana/speciali/mondo/rossi.html.

Stammerjohann H. (2010), "Italianismi", in Simone R (dir.), Enciclopedia dell'Italiano, Istituto dell'Enciclopedia Italiana, Treccani, Roma, pp. 708-711: https://www.treccani.it/enciclopedia/italianismi_(Enciclopediadell\%27Italiano)/.

Stammerjohann H. (2013), La lingua degli angeli: Italianismo, italianismi e giudiri sulla lingua italiana, Accademia della Crusca, Firenze.

Tomasin L. (2010), "Sulla diffusione del lessico marinaresco italiano", in Studi linguistici italiani, XXXVI, pp. 161-190.

Tomasin L. (2011), "La lingua della marineria", in Simone R. (dir.), Enciclopedia dell'italiano, Istituto dell'Enciclopedia Italiana, Treccani, Roma, pp. 856-858:

https://www.treccani.it/enciclopedia/lingua-della-marineria_\%28Enciclopediadell $\% 27$ Italiano $\% 29 /$.

Videsott P. (2001), "Gli italianismi nel ladino brissino-tirolese: alcuni aspetti quantitativi e cronologici in base all'ALD-I", in Linguistica, 41, 1, pp. 129-158:

https:// revije.ff.uni-lj.si/linguistica/article/view/3804/3511.

\section{Dizionari}

Cortelazzo M., Marcato C. (2005), Dį̧ionario etimologico dei dialetti italiani. UTET, Torino.

De Mauro T. (2007), Grande dizionario italiano dell'uso, con la collaborazione di Lepschy G. e Sanguineti E., UTET, Torino. 
(C) Italiano LinguaDue 2. 2021. D. Della Pietra, Gli italianismi enogastronomici recenti nella lingua inglese: un'analisi sui corpora

Gho P. $\left(2010^{2}\right)$, Dizionario delle cucine regionali italiane, Slow food, Bra.

Morris W. (1970), The American Heritage dictionary of the English language, American Heritage, New York.

Simpson J. A., Weiner (1989), The Oxford English Dictionary, Clarendon Press. E. S. C., \& Oxford University Press, Oxford.

Stammerjohann H. et alii (a cura di) (2008), Dizionario di italianismi in francese, inglese, tedesco, Accademia della Crusca, Firenze. 